\title{
Syndecans: transmembrane modulators of adhesion and matrix assembly
}

\author{
Anne Woods \\ Department of Cell Biology, University of Alabama at Birmingham, Volker Hall 203A, 1530 3rd Avenue S., \\ Birmingham, Alabama 35294-0019, USA. Phone: (205) 934-1548; Fax: (205) 975-9956; E-mail: awoods@cellbio.bhs.uab.edu.
}

Syndecans are transmembrane heparan sulfate proteoglycans (HSPGs) that are present on most cell types. HSPGs have been known for some time to regulate a variety of biological processes, ranging from coagulation cascades, growth factor signaling, lipase binding and activity, cell adhesion to ECM and subsequent cytoskeletal organization, to infection of cells with microorganisms. They are complex molecules, with specific core protein to which a variable number of glycosaminoglycan (GAG) chains are attached. Not only the number of chains varies; although syndecans mainly bear heparan sulfate GAGs, some have additional chondroitin/dermatan sulfate chains. Furthermore, heparan sulfate chains can vary in length, epimerization of glucuronic acid to iduronic acid, overall sulfation of the chains, and position of sulfation of the monosaccharides.

Early studies relied on structurally nonspecific approaches such as competition with heparin or treatment with heparinases or chlorate to remove GAG chains or prevent their sulfation, respectively. These approaches defined a number of interacting ligands for heparan sulfates including insoluble ECM components and soluble growth factors and cytokines, and they helped to implicate HSPGs in a variety of biological processes (1-5). Cell surface HSPGs, including syndecans, appear to play modulatory roles, such as presenting growth factors to their primary receptors or increasing the infectivity of viruses by interacting with their primary receptors. In addition, these molecules can modify adhesion mediated by the integrins, the major family of receptors for ECM. This last function establishes a parallel between the syndecans (as well as other cell surface HSPGs) and the secreted matricellular proteins discussed elsewhere in this Perspective series.

This is an exciting time to be in the field of syndecan research. Recently, there has been a surge of interest in the role of HSPGs and in identifying both the specific sequences of monosaccharides and sulfation patterns involved in ligand binding. With the cloning of various core proteins, it has become possible to study the features of these proteins that affect the ligand-binding ability and subsequent biological responses (reviewed in ref. 5; Table 1). Now that tools are available to analyze individual protein cores, and techniques are becoming available to determine the sequence of their GAG chains, it is becoming evident that the protein and carbohydrate components of these molecules each play specific biological roles. Recent advances in "sequencing" of GAG chains present on individual proteoglycans have begun to allow structure to be correlated with function in these complex molecules. For example, a decasaccharide containing 2-O-sulfated iduronic acid and 6-O-sulfated $\mathrm{N}$-acetylglucosamine is needed to potentiate the interaction of FGF-2 with its receptor. In contrast, a less specific sequence of seven to eight $N$-sulfated disaccharides containing iduronic acid (with or without 2-O-sulfation) serves to bind the higher affinity heparin-binding domain of fibronectin (HepII; ref. 5). This Perspective will concentrate on the syndecan family of transmembrane HSPGs and their roles in adhesion to ECM and subsequent matrix modification. In addition, it will address how syndecans may be pivotal in coordinating growth factor and adhesion signaling mechanisms. For discussion of some recent advances concerning the roles of cell surface proteoglycans in other fundamental biological processes, see refs. 2, 5, 6, and 7 and references therein.

\section{Structure of syndecans}

The core proteins of the four mammalian syndecans have divergent ectodomains, with substantial sequence conservation seen only at sites of GAG substitution. They are normally substituted with three to five heparan sulfate chains, although some members can be additionally substituted with chondroitin or dermatan sulfate. Three of the syndecans $(1,2$, and 4) have been shown to bind ECM molecules. As with growth factor binding, interactions with the ECM appear to be through the GAG chains, and since these heparan sulfate GAGs are very similar overall (reviewed in ref. 5), it might be expected that all cell surface HSPGs will bind ligands such as matrix molecules or growth factors to the same extent. Since this is clearly not the case (reviewed in ref. 1), other interactions involving regions of the core protein must affect the outcome of GAG chain binding to these ligands (1). Ligand binding also appears to be determined by specific sulfation/epimerization patterns that result in regions of high ( $S$ domains) and low ( $\mathrm{N}$ domains) modification along the 


\section{Matricellular proteins}

E. Helene Sage, Series Editor chain length. The complete sequencing of the specific GAG chains for any particular syndecan remains to be achieved but will yield extremely important information. Syndecan core proteins are small compared with their large GAG chains (1-5). These proteins contain four conserved tyrosine residues, and syndecans can be tyrosine phosphorylated, although the residues involved and the biological consequences of this modification are unclear. Their transmembrane domains are highly homologous, and their short cytoplasmic tails have two regions of high homology proximal and distal to the membrane (C1 and $\mathrm{C} 2$ ), with an intervening sequence (V region) that is specific to individual syndecans. This has led to the speculation that certain functions are common to all syndecans, with others specific for individual family members. In terms of common function, all syndecans have a COOH-terminal FYA sequence that can interact with PDZ domain containing proteins, which implies a role in protein-protein interactions. To date, three PDZ-domain proteins have been shown to interact with syndecans (Figure 1): syntenin (8), CASK/LIN (9), and synectin (10). In addition, a recently identified binding partner, synbindin (11), contains a sequence with limited homology to a PDZ domain. Initially it was thought that interactions of syndecans with PDZ domain proteins resulted in the formation of a submembraneous scaffold that connects to the cytoskeleton. However, recent evidence points to a possible role of these syndecan-binding proteins in trafficking and/or sorting of proteins to or from specific membrane areas (11-13). In particular, it appears these proteins may regulate clustering of syndecans (11). Since the FYA motif is common to all four syndecans, it might be expected that some competition for binding may occur. Syndecans, however, are highly regulated in their expression, both in development and in a cell type-specific manner. In general, syndecan-1 is the major syndecan in epithelial cells, syndecan-2 in fibroblasts, and syndecan-3 in neuronal tissue, although more than one syndecan can be expressed in the same cell type. Interestingly, syndecan-4, although a minor component, is present in a range of cell types, including fibroblasts, epithelial, and smooth muscle cells, perhaps indicating a specific role for this transmembrane proteoglycan.

The C1 domains of syndecans (Figure 1) are also highly homologous. Syndecan-3 binds the heparin-binding growth-associated molecule HB-GAM via its GAG chains. Binding results in axonal extension, concomitant with an interaction of the $\mathrm{C} 1$ domain with a complex that includes c-src and the syndecan- 3 substrate cortactin (reviewed in refs. 3-5). Cortactin interacts with the microfilament submembraneous cytoskeleton, and, perhaps indirectly, the microtubule system. Ezrin, a member of the ERM (ezrin, radixin, moesin) family, binds syndecan-2 (14). ERM proteins are also postulated to link membrane receptors to the cortical actin

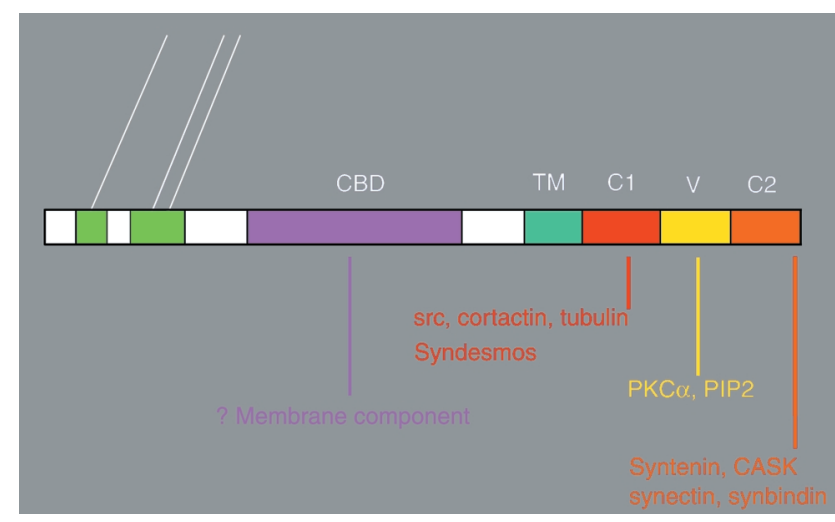

\section{Figure 1}

Schematic diagram of the known interactions of syndecan core proteins. The GAG chains on the ectodomain are indicated by white lines, and the cytoplasmic domain is divided into the $\mathrm{C} 1, \mathrm{~V}$, and $\mathrm{C} 2$ regions. Molecules known to interact with different sites within the core protein are indicated. CBD, cell binding domain; TM, transmembrane domain.

meshwork. Since previous studies indicate that ERMs bind to membrane-proximal basic amino acid sequences of transmembrane proteins, the ezrin-syndecan-2 interaction may be through the C1 domain. Again, since the C1 domains are highly conserved, it might be expected that this interaction could occur with the other syndecans. A third protein that can interact with a C1 region is syndesmos (15). Syndesmos binds the $\mathrm{C} 1$ region of syndecan-4, but here the interaction appears to be limited to syndecan-4, since the distinctive $\mathrm{V}$ region of this syndecan is also required.

The $V$ regions of syndecans are conserved between species but differ between syndecans $1-4$. Thus, the $V$ region of syndecans may determine specific biological roles. Few binding partners have been elucidated for these regions. The syndecan- $4 \mathrm{~V}$ region is required for interaction of the $\mathrm{C} 1$ region with syndesmos, but it does not seem to interact directly with this ligand, suggesting that the V-region alters the conformation of the C1 region in a way that favors the interaction with this ligand. The syndecan $-4 \mathrm{~V}$ region binds to phosphatidylinositol 4,5 bisphosphate (PIP2), as well as to the catalytic domain of the serine/threonine kinase protein kinase $\mathrm{C} \alpha(\mathrm{PKC} \alpha)$, resulting in a superactivation of this enzyme (reviewed in refs. 1-5). Superactivation of PKC $\alpha$ activity requires oligomerization of the $\mathrm{V}$ region or the whole core protein. All syndecans oligomerize to form higher-order complexes, and oligomerization may underlie their biological roles. The self-interaction of the cytoplasmic domains has only been investigated for syndecan-4 so far. Short synthetic peptides having the sequence of syndecan- $4 \mathrm{~V}$ region form dimers with a parallel twisted clamp structure (reviewed in refs. 3, 4). Dimerization is stabilized by PIP2, which promotes further oligomerization. 
Syndecans in adhesion and matrix assembly

Syndecan-1. Syndecan-1 was the first HSPG of this family to be identified and cloned. It is mainly limited to epithelial cells, but it is also found in condensing mesenchyme during development and in pre-B lymphocytes and plasma cells. Consistent with a role in adhesion, syndecan- 1 is present in a basolateral distribution in epithelia, and it appears to regulate epithelial morphology (reviewed in refs. 2, 3, 5), since transfection of epithelial cells with antisense mRNA for syndecan-1 results in an epithelial-mesenchymal switch and activates cells to invade collagen gels. The attendant loss of E-cadherin in these cells suggests a coordinate regulation of syndecan-1 and E-cadherin, and indeed, reduced E-cadherin expression can also result in decreased syndecan-1 production.

Early studies indicated that syndecan-1 may be involved in cell adhesion to ECM. Transfection of syndecan-1 into Schwann cells, which normally lack this syndecan, increases spreading and promotes the formation of focal adhesions and stress fibers. Although syndecan-1 codistributes with the microfilament system at the membrane during spreading and becomes detergent insoluble, it does not localize at focal adhesions. Indeed, there has been only one report of synde- can-1 being present in focal adhesions. Interestingly, detergent-insolubility, which is usually taken to indicate a linkage to the cytoskeleton, does not require the presence of the cytoplasmic domain; a very recent study (7) indicates that syndecan-1 transmembrane domains associate with detergent-insoluble lipid rafts as part of a specialized type of endocytosis.

Although syndecan- 1 is present in immature B cells and mature plasma cells, it is not found in B lymphocytes in the circulation, again suggesting that syndecan1 controls adhesion to ECM. Studies with myeloma cells that lack syndecan- 1 and, therefore, provide a null background for investigation of transfected constructs, indicate that syndecan- 1 does indeed increase adhesion to collagens and limit invasion of collagen gels (reviewed in refs. 1-4). Both syndecan-1 and -4 have this ability, but transfection with glypican-1 (a glycophosphoinositol-linked (GPI-linked), nontransmembrane HSPG) does not increase adhesion, suggesting that the presence of GAG chains is not sufficient for this activity and that the syndecan- 1 and -4 cytoplasmic domains might be required. However, further experiments using glypican-syndecan chimeras disproved the need for syndecan cytoplasmic domains, since the ectodomain of syndecan-1 tethered to a GPI anchor was effective.

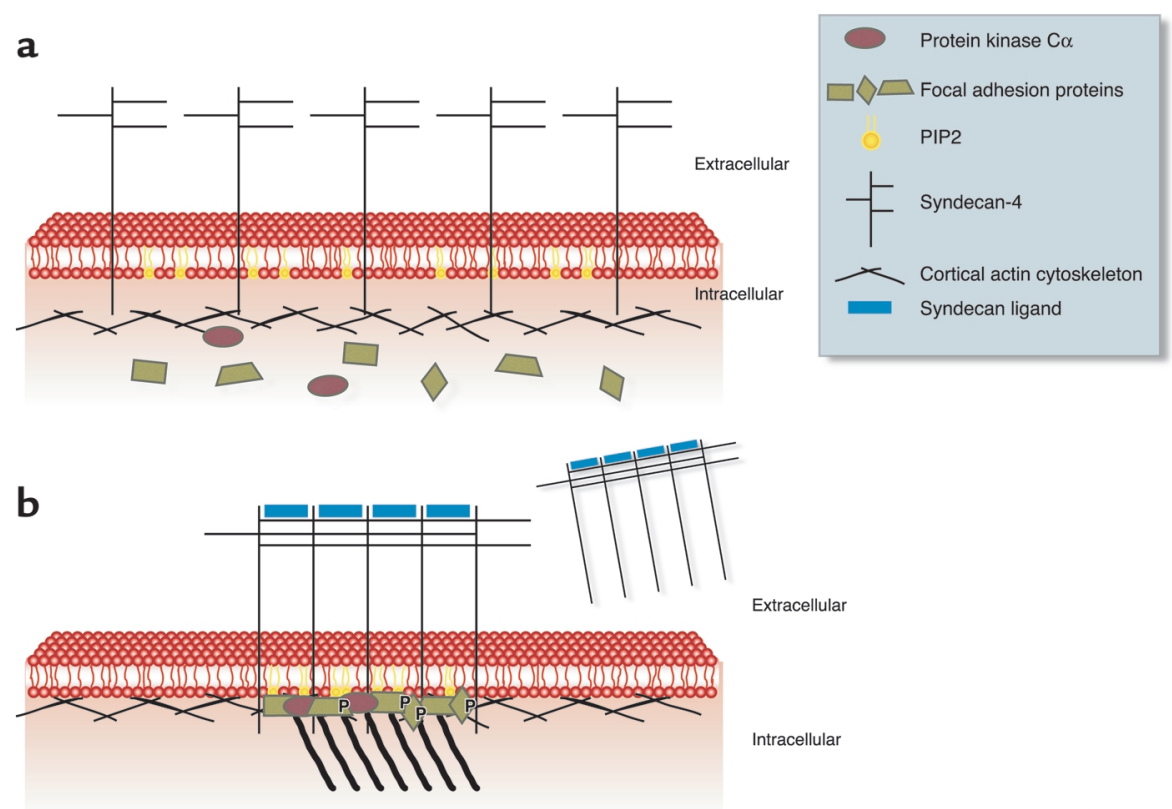

by Ken Beauchamp J. Clin. Invest.

\section{Figure 2}

Focal adhesions and stress fiber formation. (a) Syndecan-4 is diffusely distributed in the membrane, as is the phospholipid PIP2 (yellow). PKC $\alpha$ (red ovals) is cytosolic under these conditions. (b) Following interaction with ligand (blue), syndecan-4 becomes clustered into forming focal adhesions. This clustering is stabilized by PIP2 and allows binding and superactivation of PKC $\alpha$, leading to the phosphorylation of focal adhesion proteins. The actin microfilament system is condensed at focal adhesions, inducing the formation of stress fibers. Shed forms of syndecan-4 may interfere with the cell's ability to reorganize its cytoskeleton in response to adhesive interactions. The function of syndecan-4, either in its membrane-bound form or in solution, is analogous to that of a number of matricellular proteins described elsewhere in this Perspective series. 


\section{Matricellular proteins}

E. Helene Sage, Series Editor
Whether increased adhesion turns out to be due to interactions of the ectodomain or transmembrane domains of syndecan core proteins with other membrane proteins remains to be determined.

Syndecan-2. Syndecan-2 is the major syndecan in fibroblasts. Previous studies have shown that it can be phosphorylated on serine both in vivo and in vitro and that its $\mathrm{V}$ region is a substrate for PKC and PKA (reviewed in ref. 16). Syndecan-2 shows a general diffuse distribution in the membrane and does not localize to focal adhesions. However, if it is overexpressed in Chinese hamster ovary (CHO) cells, it induces increased spreading, with the cells adopting a more epithelial morphology (17). This results in an increase in stress fibers within the cells, but these do not terminate at focal adhesions. In contrast to a direct role in adhesion, syndecan-2 appears to regulate matrix deposition (16).

Formation of a pericellular matrix of fibrillar fibronectin requires the participation of activated integrins as well as syndecan-2. Transfection of CHO-K1 cells with full-length syndecan-2 has little effect on fibril assembly of endogenous fibronectin or laminin (16). However, constructs that either lack the cytoplasmic region entirely or are truncated in the middle of the $\mathrm{V}$ region inhibit fibril formation. Crucially, these mutant forms of syndecan-2 have no effect on integrin expression or the synthesis or export of fibronectin or laminin. Cells expressing truncated syndecan-2 still attach and spread on substrates of fibronectin, similar to control cells, but they fail to remodel the substrate-adsorbed fibronectin into fibrils around their edges. Similarly, soluble exogenous fibronectin was incorporated into the matrix of control cells, but not those expressing the truncated constructs. Both activation of integrins and an intact cytoskeleton are required for fibril assembly. Since fibril formation is prevented even in cells transfected with constitutively active integrins, it appears that disregulation of the cytoskeleton by the truncated syndecan- 2 is sufficient to block this process. Focal adhesions, although slightly reduced in number, appear to contain the normal repertoire of components in transfected cells, indicating that expression of the truncated syndecan- 2 may reduce fibril formation through an abnormality in the submembraneous cortical meshwork, which has been implicated in fibril nucleation and initial growth. The finding that ERM proteins may bind syndecan-2 may support a role for this HSPG in cortical microfilament function.

The molecular mechanisms underlying the lack of matrix assembly in cells carrying the truncated syndecan-2 is still under investigation, but the $\mathrm{V}$ region of syndecan-2 is a substrate for PKC, the activity of which is needed for matrix assembly (reviewed in ref. 16). Both serines in the $V$ region of syndecan- 2 can be phosphorylated, and the extent of phosphorylation is dictated by the degree of oligomerization. As discussed below, oligomerization of syndecan-4 appears to control its role in cell adhesion, so it is tempting to specu-

\section{Table 1}

Known ligands for HSPGs, where sulfation/epimerization patterns have been determined

\begin{tabular}{cc}
\hline Ligand \\
$\begin{array}{c}\text { ECM molecules } \\
\text { laminin } \\
\text { fibronectin } \\
\text { Growth factors/cytokines } \\
\text { FGF-1 and -2 } \\
\text { HGF } \\
\text { IL-8 } \\
\text { cell adhesion } \\
\text { cell adhesion } \\
\text { PDGF-AA } \\
\text { Protelet factor } 4 \\
\text { Antithrombin III }\end{array} \quad \begin{array}{c}\text { dimerization, presentation } \\
\text { to high affinity receptors } \\
\text { sequestration of growth factor } \\
\text { sequestration of cytokine }\end{array}$ \\
\hline
\end{tabular}

Details and original references can be found in ref. 5 .

late that self-association of syndecan-2 regulates matrix assembly, possibly by altering the phosphorylation status of syndecan-2. Experiments underway to transfect cells with syndecan-2 in which these serine residues are mutated will help to determine if phosphorylation of one or both serines is involved in its role in matrix assembly.

Other studies in fibroblasts have indicated possible roles for syndecan-2 in adhesion. Overexpression of syndecan-2 and syntenin increases fibroblast spreading, and the two proteins colocalize at the membrane and in intracellular vesicles (8). Similarly, Kusano et al. (18) showed that downregulation of syndecan-2 can diminish microfilament bundle formation in response to fibronectin, mimicking the effect of treatments that block heparan sulfate function. The authors concluded that syndecan- 2 and $\alpha 5 \beta 1$ integrin interactions are needed for bundle formation, a situation analogous to that seen with syndecan-4 (see below).

The other major studies on syndecan- 2 have been in the nervous system, where CASK/LIN distribution correlates with both syndecan- 2 and -3 in both a temporal and spatial manner (9). CASK, in addition to binding to the $\mathrm{C} 2$ domain (Figure 1 ) of syndecans via its PDZ domain, has a protein 4.1 binding site that could serve to link the HSPG to the cortical cytoskeleton. In transfection studies, syndecan-2 promoted the early maturation of dendritic spines in rat hippocampal neurons (19), and this required the FYA motif in the C2 region. However, truncated forms of syndecan-2 lacking the FYA motif still accumulated in the normal location, implying that the FYA motif was not needed for localization but for the subsequent biological effect. The correct distribution of syndecan-2 was lost when the cytoplasmic domain was truncated to only three amino 
acids. Hence some region of the $\mathrm{C} 1, \mathrm{~V}$, or $\mathrm{C} 2$ subdomain proximal to the FYA motif is responsible for correct trafficking and targeting.

Recent experiments have identified a new syndecan-2 binding partner, synbindin, which has a region that bears limited homology to a PDZ-domain (11). It shows greater homology with yeast proteins involved in the regulation of trafficking. Synbindin distribution is similar to that of syndecan-2 in the central nervous system, and both are concentrated in dendritic spines in mature hippocampal neurons in culture. Interestingly, the FYA sequence of syndecan- 2 is needed for clustering of synbindin in dendritic spines (11), and this region is required for accelerated spine maturation induced by syndecan-2, but not for syndecan-2 localization (19). These findings lead to the speculation that recruitment of synbindin by syndecan- 2 helps determine trafficking to maturing spines and postsynaptic structures.

Syndecan-3. This syndecan, unlike the others, appears to bind ECM components poorly. Indeed, it may function more in growth factor control of adhesion. Its heparan sulfate chains can bind HB-GAM (also termed pleiotropin), which results in axonal extension (reviewed in refs. 3, 5). Concomitant with binding is the activation of c-src and tyrosine phosphorylation of the cortical microfilament meshwork protein cortactin, leading to the formation of a complex containing syndecan-3, c-src, cortactin, and tubulin. Competition experiments indicate that the interaction is through the $\mathrm{C} 1$ domain of syndecan-3, which is intriguing in light of the homology between the $\mathrm{C} 1$ domains of the various syndecans. It has not been reported whether binding of other growth factors to other syndecans can induce the formation of similar complexes and signaling cascades, but if this response is limited to syndecan3 , it may indicate that the $\mathrm{V}$ region can modulate the interactions of $\mathrm{C} 1$ regions.

Syndecan-4. Syndecan-4 is the only syndecan that is a widespread component of focal adhesions (reviewed in refs. 2-5). Different integrins are present in focal adhesions dependent on the substrate to which the cells are attached, but syndecan- 4 is a common component irrespective of which matrix component is used. The insertion of syndecan- 4 into focal adhesions requires the activity of PKC, as does spreading and focal adhesion formation, and its overexpression in CHO-K1 cells increases spreading and results in a more fibroblastic morphology and increased formation of focal adhesions and stress fibers (17). Conversely, transfection with a truncated construct of syndecan-4 that terminates within the $V$ region reduces spreading and focal adhesion and stress fiber formation (17). Again, changes in adhesion do not appear to be through alterations in integrin levels, which indicates that, as with syndecan- 2 and matrix assembly, the cytoplasmic tail of syndecan-4 modulates integrin-mediated processes.

Biochemical experiments (reviewed in refs. 1-5) shed some light on how syndecan-4 may affect adhesion (Figure 2). Syndecan-4 fusion proteins, as well as short synthetic peptides with the sequence of the $\mathrm{V}$ region, can potentiate the activity of PKC $\alpha$ in vitro, even when the enzyme has been activated by diacylglycerol and phosphatidylserine. This effect requires oligomerization of the fusion proteins or peptides, and minor substitutions in peptide sequence abolish both potentiation and oligomerization. Similarly, PIP2, which alone can partially activate $\mathrm{PKC} \alpha$, augments oligomerization, and a ternary mixture with syndecan-4 results in an enzymatic activity higher than that seen with normal mediators. The presence of syndecan-4 and PIP2 also results in the conventional PKC $\alpha$ isoform becoming calcium-independent. PKC can be coclustered with syndecan-4 in live cells, and both molecules are found as components of focal adhesions. In addition, $\mathrm{PKC} \alpha$ can be both coimmunoprecipitated with syndecan-4 and can bind to syndecan-4 peptide in vitro. However, the presence of a ternary complex has not yet been identified in vivo. A ternary complex may be important since many of the focal adhesion or cortical meshwork components are substrates for $\mathrm{PKC} \alpha$ and have their conformation altered by PIP2. Integrin ligation increases the levels of PIP2, which may stabilize syndecan-4 oligomers induced by interaction with heparin-binding domains of matrix molecules, thus allowing PKC $\alpha$ to bind and phosphorylate specific components at nascent focal adhesions.

Early studies that indicated a need for both integrin ligation and an interaction with the heparin-binding domain of fibronectin for stress fiber and focal adhesion formation, have recently been confirmed and extended (3). One study showed that, although fibronectin-null fibroblasts can attach and spread on recombinant fragments of fibronectin that contained the RGD integrinbinding sequence, they fail to form focal adhesions unless triggered by addition of heparin-binding domains or by the clustering of syndecan-4 with antibody. Pharmacological activation of Rho GTPase can circumvent the need for heparin-binding domains or antibody clustering, whereas its inhibition prevented the induction of focal adhesion formation. Another study confirmed that $\mathrm{CHO}$ cells that are incapable of glycanating proteoglycans due to an enzyme defect lack focal adhesions unless syndecan-4 is overexpressed, which may force the oligomerization of the syndecan-4 core proteins in the absence of heparan sulfate chains. Finally, fibroblasts from mice that lack syndecan- 4 cannot respond to the heparin-binding region of fibronectin when spread on the integrin-binding fragment (20). They do, however, respond to intact fibronectin, implying that sufficient concentration, or a difference in presentation, may influence biological activity.

Although syndecan-4 knockout mice appear normal, they do, as with syndecan-1 knockout mice, show defects in wound healing (21), where responses to 


\section{Matricellular proteins}

E. Helene Sage, Series Editor growth factors and ECM regulate migration, division, and wound closure. Since transfection with different syndecan constructs result in such different effects (e.g., on adhesion or on matrix assembly), it is difficult to envisage how syndecan family members may substitute for each other, although this possibility has not been tested. Growth factor stimulation may circumvent signaling through syndecans and, indeed, treatment with some growth factors can promote focal adhesion formation in cells on the integrin-binding fragment of fibronectin. These observations lead to a final interesting concept: Syndecans are involved in both growth factor binding and interaction with ECM molecules. It is now becoming clear that signaling from adhesion through integrins and signaling through growth factor binding can converge to coordinate cellular responses. Thus, in the absence of one set of signals, the other may circumvent the missing cascades.

Three separate indications of convergence of signaling through syndecans on adhesion- and growth factor-mediated responses should be highlighted. First, serum-starved quiescent $3 \mathrm{~T} 3$ cells show phosphorylation of the serine in the $\mathrm{C} 1$ region of syndecan- 4 . This modification, which can be reversed rapidly upon addition of FGF-2, controls syndecan-4 oligomerization, interaction with PIP2, and ultimately the ability to activate PKC $\alpha$ (reviewed in refs. 3-5). Second, syndecan-3 binding to HB-GAM initiates a src-mediated signaling cascade, concomitant with a trigger for axonal extension. Third, cells lacking focal adhesions when spread on the integrin-binding fibronectin can be prevented from responding to heparin-binding domains of fibronectin via syndecan-4 by inhibition of the GTPase Rho. The family of Rho, Rac, Cdc42 and Ras GTPases is often involved downstream of many signaling cascades resulting from growth factors or adhesion and can regulate cytoskeletal organization, thus determining morphology. Furthermore, syndecans can be shed from the cell surface, effectively providing a soluble competitor for cell surface syndecans, which not only retain capacity to bind growth factors, but also can regulate protease activity, thus affecting matrix stability $(2,3,5)$. The ability of cell surface or soluble syndecans to participate in a range of fundamental metabolic activities endows these molecules with broad regulatory roles in cell-matrix and cell-growth factor interactions.

\section{Implications for disease}

The pathological roles of syndecan have not been extensively studied, partly due to a lack of specific tools, such as antibodies that recognize core proteins. However, many studies are now ongoing, and a few are beginning to be reported in the literature (reviewed in ref. 5). Thus, during wound healing, both syndecan-1 and syndecan-4 are increased in expression, and in their cleavage and release in soluble form. The expression of some growth factors, such as FGF-1, -2 and -7 , is also increased in wound healing, and these factors require HSPG for full activity. Increased expression of syndecan- 1 and -4 is seen during dermal and mucosal repair, and during response to arterial injury. Mice deficient in syndecan- 1 show delayed wound healing. Furthermore, since fibrosis can be thought of as misregulated wound healing in which deposition progresses too far, too early, or with the wrong set of proteins, the effects of syndecan- 2 on matrix assembly, described above, may well contribute to many diseases involving a fibrotic response.

Roles of syndecans in tumorigenesis are also under investigation. Tumorigenesis is associated with the loss of normal cell morphology, a gain of invasiveness, decreased adhesion, and abnormal proliferation control. Syndecans have been implicated in the regulation of all these fundamental processes. Syndecan- 1 is reduced when epithelial cells are transformed, and several studies now indicate that its decrease may be a prognostic marker in some tumors. Interestingly, a recent study of mice transgenic for Wnt-1 showed that they had increased levels of mammary tumor formation. However, if the mice were additionally syndecan1 deficient, tumor formation was reduced (see ref. 6 and references therein).

\section{Conclusions}

Syndecans participate in a range of fundamental biological processes. They are unique in that their GAG chains allow interactions with ECM proteins, growth factors, metalloproteinases, lipases, and even pathogens. Improved sequencing techniques have led to the concept of highly specific interaction sites within regulated domains along the polysaccharide chains. It will be fascinating to discover whether the GAG chains of individual syndecans contain differing microdomains that regulate specific interactions. Syndecans not only function at the cell surface, but, since their shed ectodomains retain function, they may be a type of matricellular protein (22) that regulates both growth factor and matrix interactions.

In addition to the ligand-binding GAG chains, the transmembrane core proteins of syndecans can also have biological activity. Some signaling functions of syndecans have already been identified and more are sure to follow. Those identified to date confirm the regulatory roles of these molecules, in that they modify signaling cascades initiated by primary receptors or increase ligand interaction with primary receptor. So far, most core protein functions have been attributed to the cytoplasmic domain, but there is increasing evidence for a role for their ectodomains in adhesion. The next few years should be interesting, with increased interest in the field leading to new discovery. Do cell surface syndecans physically associate with other surface molecules, such as integrins or growth factor 
receptors, to modulate activity? If so, are the interaction sites extracellular, transmembrane, or intracellular? Preliminary data (23) indicate association of syndecan-4 with both cytoskeletal components and integrins, and future studies on how these interactions are controlled in development and disease will also be an exciting area of research.

\section{Acknowledgments}

I would like to thank friends and colleagues for useful discussions. This work was supported by NIH grant DK 54605.

1. Rapraeger, A.C. 2000. Syndecan-regulated receptor signaling. J. Cell Biol. 149:995-997.

2. Bernfield, M., et al. 1999. Functions of cell surface heparan sulfate proteoglycans. Annu. Rev. Biochem. 68:729-778.

3. Couchman, J.R., Chen, L., and Woods, A. 2001. Syndecans and cell adhesion. Int. Rev. Cytol. In press.

4. Woods, A., and Couchman, J.R. 2000. Integrin modulation by lateral association. J. Biol. Chem. 275:24233-24236.

5. Tumova, S., Woods, A., and Couchman, J.R. 2000. Heparan sulfate proteoglycans on the cell surface: versatile coordinators of cellular functions. Int. J. Biochem. Cell Biol. 32:269-288.

6. Alexander, C.M., Hinkes, M.T., and Bernfield, M. 2000. Syndecan-1 is required for Wnt-1 induced tumorigenesis but not for morphogenesis of mouse mammary epithelia. Nat. Genet. 25:329-332.

7. Fuki, I.V., Meyer, M.E., and Williams, K.J. 2000. Transmembrane and cytoplasmic domains of syndecan mediate a multi-step endocytic pathway involving detergent-insoluble membrane rafts. Biochem. J. 351:607-612.

8. Grootjans, J.J., et al. 1997. Syntenin, a PDZ protein that binds syndecan cytoplasmic domains. Proc. Natl. Acad. Sci. USA. 94:13683-13688.

9. Hsueh, Y.P., et al. 1998. Direct interaction of CASK/LIN-2 and syndecan heparan sulfate proteoglycan and their overlapping distribution in neu- ronal synapses. J. Cell Biol. 142:139-151.

10. Gao, Y., Li, M., Chen, W., and Simons, M. 2000. Synectin, syndecan-4 cytoplasmic domain binding PDZ protein, inhibits cell migration. J. Cell. Physiol. 184:373-379.

11. Ethell, I.M., Hagihara, K., Miura, Y., Irie, F., and Yamaguchi, Y. 2000. Synbindin, a novel syndecan-2-binding protein in neuronal dendritic spines. J. Cell Biol. 151:53-67.

12. Fialka, I., et al. 1999. Identification of syntenin as a protein of the apical early endocytic compartment in Madin-Darby canine kidney cells. J. Biol. Chem. 274:26233-26239.

13. Wang., L.-H., Kalb, R.G., and Strittmatter, S.M. 1999. A PDZ protein regulates the distribution of the transmembrane semaphorin, M-SemF.J. Biol. Chem. 274:14137-14146.

14. Granes, F., Urena, J.M., Rocamora, N., and Vilaro, S. 2000. Ezrin links syndecan-2 to the cytoskeleton. J. Cell Sci. 113:1267-1276.

15. Baciu, P.C., et al. 2000. Syndesmos, a protein that interacts with the cytoplasmic domain of syndecan-4, mediates cell spreading and actin cytoskeletal association. J. Cell Sci. 113:315-324.

16. Klass, C.M., Couchman, J.R., and Woods, A. 2000. Control of extracellular matrix assembly by syndecan-2 proteoglycan. J. Cell Sci. 113:493-506.

17. Longley, R.L., et al. 1999. Control of morphology, cytoskeleton and migration by syndecan-4. J. Cell Sci. 112:3421-3431.

18. Kusano, Y., et al. 2000. Participation of syndecan 2 in the induction of stress fiber formation in cooperation with integrin $\alpha 5 \beta 1$ : structural characteristics of heparan sulfate chains with avidity to COOH-terminal heparin-binding domain of fibronectin. Exp. Cell Res. 256:434-444.

19. Ethell, I.M., and Yamaguchi, Y. 1999. Cell surface heparan sulfate proteoglycan syndecan- 2 induces the maturation of dendritic spines in rat hippocampal neurons. J. Cell Biol. 144:575-586.

20. Ishiguro, K., et al. 2000. Syndecan-4 deficiency impairs focal adhesion formation only under restricted conditions. J. Biol. Chem. 275:5249-5252.

21. Echtermeyer, F., et al. 2001. Delayed wound repair and impaired angiogenesis in mice lacking syndecan-4. J. Clin. Invest. 107:R9-R14.

22. Bornstein, P. 1995. Diversity of function is inherent in matricellular proteins: an appraisal of thrombospondin. J. Cell Biol. 130:503-506.

23. Greene, D.K., Woods, A., Tumova, S., and Couchman, J.R. 2000. Syndecan-4 associations with the microfilament cytoskeleton and integrins. Mol. Biol. Cell 11:349a. (Abstr.) 